Revue d'histoire de l'Amérique française

REYUE D.HISTOIRE DE L'AMÉRIQUE FRANÇAISE

\title{
Sur quelques bibliographies récentes
}

\section{Paul-André Linteau}

Volume 28, numéro 1, juin 1974

URI : https://id.erudit.org/iderudit/303332ar

DOI : https://doi.org/10.7202/303332ar

Aller au sommaire du numéro

Éditeur(s)

Institut d'histoire de l'Amérique française

ISSN

0035-2357 (imprimé)

1492-1383 (numérique)

Découvrir la revue

\section{Citer cette note}

Linteau, P.-A. (1974). Sur quelques bibliographies récentes. Revue d'histoire de l'Amérique française, 28(1), 105-112. https://doi.org/10.7202/303332ar d'utilisation que vous pouvez consulter en ligne.

https://apropos.erudit.org/fr/usagers/politique-dutilisation/ 


\section{Sur quelques bibliographies récentes}

La bibliographie historique a connu un essor remarquable au Québec depuis quelques années. Comme si une science historique en effervescence avec des effectifs en croissance rapide, orientée sur une foule de pistes nouvelles voulait éviter de faire tout à fait table rase et sentait le besoin de s'élancer non pas de la rive, mais du tremplin de l'histoire déjà constituée. Les grands projets de recherche historique lancés depuis quelque temps au Québec ont tous été l'occasion d'efforts bibliographiques importants. En même temps qu'ils ouvraient des pistes nouvelles, les jeunes historiens québécois redécouvraient un grand nombre de travaux laissés dans l'oubli par la génération précédente, une génération qui à cause de la faiblesse de ses effectifs avait dû négliger de vastes pans de l'histoire québécoise.

Le Québec a, dans le domaine, des traditions. Les Gagnon, Dionne et Malchelosse, bibliographes solitaires compilant patiemment, au fil des ans, des listes considérables, ont, en leur temps, bien mérité de la patrie. Ils ont encore aujourd'hui un disciple en la personne de Claude Thibault. Mais c'est là une tradition qui se perd. Personne d'ailleurs ne s'en plaindra car la bibliographie québécoise est plus vivante que jamais, et peut-être aussi plus près des préoccupations des historiens contemporains. Les solitaires ont cédé la place aux équipes, dont la taille va du tandem à la dizaine. A côté des bibliographies générales se sont multipliés les travaux spécialisés qui collent de plus près aux besoins actuels de la recherche et, disons-le, aux modes du jour.

Quelques-uns de ces travaux font l'objet du présent commentaire. A tout seigneur, tout honneur, commençons par Beaulieu et Hamelin, ${ }^{1}$ ces chefs de file de la bibliographie québécoise. On sait l'importance de leurs travaux. L'histoire générale du Canada, les journaux et les publications gouvernementales ont tour à tour fait l'objet de leurs investigations. Jean Hamelin a raconté l'historique de cette démarche. ${ }^{2}$ Le dernier ouvrage

${ }^{1}$ André Beaulieu et Jean Hamelin, La Presse québécoise des origines à nos jours. Tome premier: 1764-1859 (Québec, PUL, 1973), $268 \mathrm{p}$.

2 Jean Hamelin, "Un catalogue des périodiques au Canada français. Genèse de quelques instruments de travail", Recherches sociographiques, VIII, 2 (mai-août 1967) : 211-213.

RHAF, vol. 28, no 1 (juin 1974) 
paru fait suite aux Journaux du Québec de 1764 à 1964 publié en 1965. Les auteurs ont élargi leur perspective en ajoutant aux journaux les autres périodiques qui constituent une part importante de La Presse québécoise: c'est là une heureuse innovation. Ils ont aussi profité de la nouvelle édition pour corriger et compléter la première à la lumière des réactions de nombreux utilisateurs. Pour chaque périodique on trouve, quand ils sont disponibles, les mêmes renseignements: le titre et ses variations dans le temps, le lieu de publication, les années de parution, la périodicité, la position politique, le format, la localisation des originaux et des microfilms, les noms des fondateurs, propriétaires, rédacteurs et des principaux auteurs. Le tout est suivi d'une notice historique dont la longueur varie avec l'importance du journal.

Les inscriptions ont été classées par ordre chronologique de fondation des périodiques. Cette méthode est discutable. L'objectif est de grouper ensemble les journaux d'une même époque. La méthode atteint son but dans le cas des journaux éphémères qui ont foisonné au Québec, mais elle est faussée dans le cas de ceux dont l'existence s'étend sur plusieurs décennies. Heureusement, un excellent index des titres et des noms d'individus facilite la tâche de l'utilisateur qui ignore la date de fondation. On ne peut que regretter cependant l'absence de la dimension spatiale qui constituait pourtant l'armature de l'édition précédente; souhaitons que les prochains tomes soient dotés d'un index des noms de lieux de publication.

La dimension la plus originale du travail de Beaulieu et Hamelin, quand on le compare aux autres compilations du genre, réside dans les notes historiques. Elles permettent de se faire rapidement une idée de l'orientation d'un journal et des politiques qu'il défend. Elles laissent cependant le lecteur sur sa faim puisqu'il s'agit de points de départ plutôt que d'analyses détaillées; ces dernières auraient nécessité des recherches d'une telle ampleur qu'il eût été impossible de les réaliser en quelques années. Ces notes présentent toutefois une lacune dans le cas des journaux qui, nés au $19 \mathrm{e}$ siècle, ont poursuivi leur carrière au $20 \mathrm{e}$ siècle: leur histoire récente fait l'objet d'un traitement trop rapide. 
Abordons un dernier point, d'ordre technique. La qualité de la présentation matérielle s'est détériorée entre l'édition de 1969 et celle de 1973. Dans le premier cas la composition typographique permettait un jeu de caractères facilitant la consultation. Le procédé choisi pour La Presse québécoise rend beaucoup plus laborieux le repérage des informations.

Malgré ces quelques lacunes La Presse québécoise est indéniablement un ouvrage de qualité, un instrument de travail conçu d'abord et avant tout pour les historiens et bien adapté à leurs besoins.

Passons maintenant au travail beaucoup plus ambitieux de Claude Thibault. ${ }^{3}$ Sa Bibliographia Canadiana rassemble 25,660 titres de livres et d'articles sur l'histoire du Canada, classés par périodes et par sujets. Sa parution réjouira tous les historiens car l'absence d'un tel ouvrage de référence se faisait réellement sentir.

L'information de Claude Thibault est très étendue. La liste des abréviations comprend environ 650 titres de périodiques. L'ampleur du matériel recueilli étonne. Devant un tel travail, on serait d'abord tenté d'offrir des félicitations - d'ailleurs bien méritées - à l'auteur et de fermer les yeux sur les imperfections qui s'y seraient glissées ici et là. Mais en manipulant l'ouvrage, on découvre des défauts qu'il est difficile de garder sous silence, d'autant plus qu'avec sa présentation luxueuse l'ouvrage n'a rien d'une édition de rodage. Le caractère plutôt définitif du travail et le prix $(\$ 25.00)$ que doit débourser l'acheteur incitent ce dernier à se montrer plus exigeant.

Le compilateur a voulu être exhaustif. Il déclare: "Bibliographia Canadiana - ou, tout ce que vous voulez connaître de l'histoire du Canada et étiez incapables de retrouver - se targue de contenir presque tous les livres publiés avant 1970 et presque tous les articles de périodiques d'avant 1969." Voilà un objectif bien téméraire et il serait illusoire d'espérer l'atteindre sans l'aide d'une équipe multidisciplinaire disposant de puissants moyens. On pourrait faire une longue énumération de titres oubliés, depuis la "Bibliographie" de la $R H A F$ jusqu'aux nombreuses études d'histoire de la médecine publiées dans l'Union

${ }^{3}$ Claude Thibault, Bibliographia Canadiana (Don Mills, Ont., Longman Canada, 1973), LXIV - $795 \mathrm{p}$. 
Médicale du Canada. En outre l'auteur n'a pas retenu les thèses et ne s'explique même pas sur cette exclusion fort discutable.

Dans le cas des livres, Claude Thibault omet trois renseignements bibliographiques utiles: la maison d'édition, le nombre de pages et la mention de texte polycopié quand c'est le cas. Cette situation ne rend que plus difficile la tâche du chercheur. Prenons à titre d'exemple, le no 287, un travail de J.-P. Chalifoux, Bibliographie sur des questions actuelles. Thibault nous indique seulement sa parution à Montréal en 1968. Or, il s'agit d'un texte polycopié à tirage restreint, réalisé par le Centre d'Etudes canadiennes-françaises de McGill. Montréal étant une grande ville nous souhaitons beaucoup de succès à celui qui voudra se procurer ce travail sans connaître ces coordonnées. De même l'absence du nombre de pages ne permet pas de distinguer les brochures des volumes.

Une autre déficience de l'ouvrage se situe au niveau de l'index. Celui-ci ne comprend pas les noms des auteurs. Les individus n'y sont mentionnés que lorsqu'ils sont les sujets d'un article ou d'un volume. Cette lacune accrô̂t la difficulté de consultation de cette bibliographie.

Bibliographia Canadiana n'en est pas moins un travail impressionnant. Son utilisation reste toutefois une opération parsemée d'embûches à la suite des choix malheureux au plan méthodologique qu'a faits le compilateur ou son éditeur. Souhaitons qu'une seconde édition fournisse bientôt l'occasion de polir le style de la version française de la préface et de l'introduction, car il en a sérieusement besoin.

Signalons au passage une autre bibliographie générale, celle de Jacques Cotnam ${ }^{4}$, qui se classe dans une catégorie différente. Destinée spécifiquement aux "English-speaking highschool and college students", elle permet un survol général et assez rapide de la production récente dans des domaines variés, depuis la religion et le folklore jusqu'au nationalisme et au syndicalisme. Le public auquel elle s'adresse y trouvera un bon point de départ. L'ouvrage sera moins utile aux Québécois qui peuvent trouver ailleurs des inventaires plus détaillés.

4 Jacques Cotnam, Contemporary Quebec: an analytical bibliography (Toronto, McClelland and Stewart, 1973), $112 \mathrm{p}$. 
Avec Le Monde Ouvrier au Québec ${ }^{5}$ de Leblanc et Thwaites, nous revenons à la bibliographie spécialisée. Ce livre témoigne de l'essor rapide qu'ont connu depuis quelques années, les recherches sur l'histoire des travailleurs québécois. Sans prétendre être exhaustif il fournit un premier instrument de travail aux chercheurs en ce domaine. Avec ses 2,927 titres il dépasse en ampleur des travaux bibliographiques réalisés jusqu'ici sur le même sujet. Ce repérage de l'information s'avérait d'autant plus indispensable que les sources de l'histoire des travailleurs diffèrent nettement de celles des objets d'étude habituellement privilégiés des historiens: elles sont plus difficiles à saisir et se retrouvent moins souvent dans les publications "historiques", que dans les revues de combat ou les publications ayant un caractère assez général.

Les auteurs ont voulu leur bibliographie ouverte à toutes les facettes du monde du travail. Au-delà des conditions de travail et du syndicalisme ils ont voulu rejoindre "le milieu social" dans lequel s'insère le travailleur. Il est question de vie urbaine (pourquoi y classer les orphelinats agricoles $(1,464)$ et l'électrification rurale $(1,465)$ ?), de niveau de vie, de logement, de santé, des mouvements de population, de l'histoire des villes et de la société en général. On rejoint ainsi l'histoire sociale, l'histoire globale.

Si tout le vécu - y compris l'histoire des patrons (et de leurs institutions) qui les dominent - concerne les travailleurs, qu'est-ce qui distingue leur histoire de l'histoire globale et pourquoi cette appellation particulière ? Il y a là un problème de définition que les auteurs ont éludé et sur lequel il faudra bien qu'ils se prononcent un jour.

Une section importante est consacrée à l'action politique ouvrière. A la fois neuve et utile, elle a toutefois une allure un peu trop fourre-tout et les auteurs prennent peut-être lours rêves pour des réalités en associant tous les mouvements de gauche au Québec à l'histoire ouvrière. La section sur la presse ouvrière est, elle aussi, neuve et susceptible de rendre de précieux services aux historiens. La mention des thèses déposées dans les universités constitue aussi un actif pour la bibliographie.

Nous sommes en présence de la première version d'un outil qui prendra une forme plus définitive quand les recherches

5 André $\mathrm{E}$. Leblanc et James D. Thwaites, Le monde ouvrier au Québec. Bibliographie rétrospective (Montréal, PUQ, 1973), $283 \mathrm{p}$. 
auront progressé et permis d'étoffer cette bibliographie. Tout inachevé que soit cet instrument de travail, il rend déjà de précieux services. Il importait de le rendre disponible, même dans cette édition de rodage.

On peut le compléter en consultant son pendant anglo-canadien ${ }^{6}$, œuvre d'une équipe de jeunes historiens. Ce livre relève 3,347 titres. Une partie substantielle est consacrée aux sources manuscrites dans les dépôts d'archives. On y trouve en outre des titres de journaux, de brochures et de publications gouvernementales. La méthode de classement laisse à désirer: un regroupement par sujets aurait probablement été plus utile. L'ouvrage est riche d'informations aussi bien sur le monde du travail que sur l'histoire des entreprises en général. On le lira avec profit.

Le dernier ouvrage sur lequel nous nous pencherons, celui de Benoît-Beaudry Gourd 7, représente un autre type de bibliographie spécialisée, centrée sur la région. Sa parution s'inscrit elle aussi dans le contexte de l'évolution récente de l'historiographie québécoise. Quelques années après les autres pays, le Québec découvre à son tour la perspective régionale dans les études historiques. Le développement des études collégiales, puis universitaires, dans les régions périphériques n'est pas étranger à cet état de choses. Depuis toujours l'apanage des amateurs, l'histoire régionale devient maintenant l'affaire des professionnels qui y introduisent une méthodologie et des perspectives inconnues jusqu'ici.

L'Abitibi-Témiscamingue, région que l'on dit ignorée au profit des grands centres, a tout de même fait parler d'elle puisque Benoît-Beaudry Gourd a relevé 2,787 titres. Nous voilà en présence d'un pays minier: rien d'étonnant à ce que $40 \%$ des titres soient consacrés à ce sujet. Les travaux des géologues y tiennent une place de choix, car la perspective du compilateur n'est pas qu'historique; il a tenté de cerner la complexité de la région dans ses dimensions passées et présentes.

${ }^{6}$ Russel G. Hann, Gregorv S. Kealev, Linda Kealev et Peter Warrian, Primary Sources in Canadian Working Class History, 1860-1930 (Kitchener, Dumont Press, 1973), 169 p.

7 Benoît-Beaudry Gourd, Bibliographie de l'Abitibi-Témiscamingue (Rouyn, Université du Québec, Direction des Etudes universitaires dans l'Ouest québécois, Nord-Ouest 1973), $270 \mathrm{p}$. 
L'ouvrage est le résultat d'un effort de cueillette systématique non seulement des livres et articles mais aussi des thèses et surtout des textes polycopiés qui sont l'émanation des groupes de pression et des associations diverses. La localisation des textes autres que les articles est indiquée. Les descriptions bibliographiques sont très bien faites et orientent adéquatement le lecteur.

Le classement laisse toutefois à désirer. On a placé une section "histoire" alors qu'il y a des travaux historiques dans les autres sections. Les ouvrages généraux côtoient des monographies très spécialisées. L'addition de quelques divisions supplémentaires au sein des chapitres aurait facilité la consultation. Ajoutons cependant que d'excellents index et un système de renvoi (pour les auteurs et les sujets) permettent de minimiser cette difficulté.

$\mathrm{Au}$ total, Benoît-Beaudry Gourd a produit un ouvrage de qualité qui, même en édition préliminaire, ne manque pas d'impressionner. Tous les lecteurs souhaiteront que chaque région du Québec soit bientôt dotée d'un semblable instrument de travail.

Ce survol rapide permet de mieux voir où va la bibliographie québécoise. A cause de l'ampleur de ses objectifs le cas de Bibliographia Canadiana est exceptionnel. La tendance est plutôt, maintenant, à la bibliographie spécialisée. Un grand nombre de travaux du genre sont parus depuis quelques années. ${ }^{8}$

Cette méthode permet de fouiller beaucoup plus en profondeur une question et de fournir aux chercheurs une information considérable.

Un autre aspect de ces travaux mérite d'être signalé. Les compilateurs faisaient face à un dilemme: recueillir patiemment, pendant des années, des informations afin de publier un ouvrage définitif ou lancer rapidement une version préliminaire, qu'ils savaient incomplète en espérant la compléter et l'améliorer un jour. Ils ont, et avec raison, choisi la deuxième solution. Ce faisant, ils répondaient aux besoins pressants de la recherche et

8 Pensons aux bibliographies publiées par le Centre d'Etudes canadiennes-françaises ou à celles de l'Institut supérieur des Sciences humaines. Voir le compte rendu de Yvan Lamonde, dans la RHAF, 27, 3 (décembre 1973) : 432-435. 
faisaient du bibliographe un homme à l'écoute de l'historien et des autres chercheurs. Ils ont aussi battu en brèche la tendance des historiens québécois à vouloir vider les questions d'un seul coup; ils ont en effet introduit la notion du construit successif enrichissant à chaque étape le travail de base. Ils ont remplacé la notion de propriété privée des sources d'information, chère au chercheur solitaire, par celle de diffusion et de mise en commun, appelant l'ensemble des chercheurs à collaborer à l'amélioration de l'œuvre.

S'il est un reproche que l'on peut adresser à ces compilateurs, c'est d'avoir parfois négligé de s'expliquer en introduction sur la nature et les limites du sujet et sur les choix méthodologiques et bibliographiques. Cette situation peut certainement être corrigée dans les éditions futures.

Entre-temps, les lecteurs seront bien avisés de communiquer aux compilateurs leurs commentaires. De cette façon, toute la communauté scientifique participera à la mise au point d'instruments de travail de qualité.

Département d'histoire

Université du Québec à Montréal

PAUL-ANDRÉ LINTEAU 